Article

\title{
Ablation of Left Atrial Tachycardia following Catheter Ablation of Atrial Fibrillation: 12-Month Success Rates
}

\author{
Armin Luik ${ }^{1, *}$, Kerstin Schmidt ${ }^{1}$, Annika Haas ${ }^{1}$, Laura Unger ${ }^{2} \mathbb{D}$, Panagiotis Tzamalis ${ }^{1}$ \\ and Bernd Brüggenjürgen ${ }^{3}$
}

check for updates

Citation: Luik, A.; Schmidt, K.; Haas, A.; Unger, L.; Tzamalis, P.; Brüggenjürgen, B. Ablation of Left Atrial Tachycardia following Catheter Ablation of Atrial Fibrillation: 12-Month Success Rates. J. Clin. Med. 2022, 11, 1047. https://doi.org/ $10.3390 /$ jcm 11041047

Academic Editor: Amir Jadidi

Received: 31 December 2021

Accepted: 15 February 2022

Published: 17 February 2022

Publisher's Note: MDPI stays neutral with regard to jurisdictional claims in published maps and institutional affiliations.

Copyright: (C) 2022 by the authors. Licensee MDPI, Basel, Switzerland. This article is an open access article distributed under the terms and conditions of the Creative Commons Attribution (CC BY) license (https:// creativecommons.org/licenses/by/ $4.0 /)$
1 Karlsruhe Municipal Hospital, Academic Teaching Hospital of the University of Freiburg, 76133 Karlsruhe, Germany; kerstin.schmidt@klinikum-karlsruhe.de (K.S.); annika.haas@klinikum-karlsruhe.de (A.H.); panagiotis.tzamalis@klinikum-karlsruhe.de (P.T.)

2 Institute of Biomedical Engineering, Karlsruhe Institute of Technology, 76131 Karlsruhe, Germany; laura.unger@kit.edu

3 Institute for Health Services Research and Technical Orthopaedics, Hanover Medical School, 30625 Hannover, Germany; bernd.brueggenjuergen@t-online.de

* Correspondence: armin.luik@klinikum-karlsruhe.de; Tel.: +49-721-9740

\begin{abstract}
The treatment of atrial tachycardia following catheter ablation of atrial fibrillation is often challenging. Electrophysiological studies using high-resolution 3D mapping systems have contributed significantly to their understanding, and new ablation approaches have shown high rates of acute terminations with low recurrences for the clinical AT. However, patient populations are very heterogeneous, and long-term data of the freedom from any atrial tachycardia or any arrhythmia are still sparse. To evaluate long-term success, a unified patient population and predefined ablation strategies are preferred. In this study, we present 12-month success and mean 30 month follow-up data of catheter ablation of left atrial tachycardia. All 35 patients had a history of pulmonary vein isolation (PVI), 71\% of which had a previous substrate modification. A total of 54 ATs, with a mean cycle length $297 \pm 86$ ms, 31 macro-reentries, and 4 localized reentries, were targeted. The ablation strategy to be used was given by the study protocol, depending on the type of reentry and the number of critical isthmuses. All available ablation strategies were included: standard (anatomical) lines, individual lines, critical isthmuses, and focal ablation. All ATs were terminated by ablation. A total of $91 \%$ terminated upon the first ablation strategy. Freedom from any AT after 12 months was $82 \%$, and from any arrhythmia, it was $77 \%$. The multi-procedure success after 30 months was $65 \%$ for any AT and 55\% for any arrhythmia. In conclusion, individual ablation strategies based on the reentry mechanism and the number of critical isthmuses seems promising and demonstrates a high long-term clinical success. Tachycardia comprising a single critical isthmus can be ablated by critical isthmus ablation only. These patients present with the highest 12-month and long-term success rates.
\end{abstract}

Keywords: atrial tachycardia; atrial flutter; catheter ablation; critical isthmus; long term success

\section{Introduction}

The crucial mechanism of atrial tachycardia (AT) can be very diverse. The incidence is age- and gender-dependent, with 5/100,000 in patients under 50 years and approximately $600 / 100,000$ in subjects $>80$ years of age $[1,2]$. Left atrial tachycardia most commonly occurs after previous cardiac surgery or catheter ablation of atrial fibrillation (AF), but it can also be idiopathic. The reported prevalence of AT after AF catheter ablation (CA) ranges from $2 \%$ to $20 \%$ [3-5] and is mostly associated with structural heart disease [3]. Although external cardioversion $(\mathrm{CV})$ and medical rate control are recommended by the recent ACC/AHA/HRS guidelines for acute treatment, relapse rates after CV are high [6]. CA offers a promising, however, challenging and time-consuming, alternative. Reported acute success rates of CA, mostly defined as restoring the sinus rhythm (SR) rather than noninducibility of arrhythmias, vary between $73 \%$ and $93 \%$ [6-15]. Reported recurrence rates 
for AT are clinically relevant and vary considerably between $7 \%$ and $53 \%[6,8,10-16]$ with one study reporting an average time to recurrence of 49 days. Focal firing, spiral excitation patterns, and reentry were found to be driving mechanisms of ATs. Reentry, mainly named as atrial flutter, is the most frequently observed mechanism and can be subdivided into macro- and micro-reentry mechanisms $[17,18]$. The complexity and variability of these circuits is related to the presence of zones of block, slow conduction, and electrically silent areas. 3D electro-anatomical mapping systems have improved the understanding of complex AT mechanisms while facilitating mapping as well as guiding ablation [19-21]. However, depending on the alterations of the underlying substrate, especially the presence of areas of a very low voltage, the interpretation of the AT may be misleading, even by the use of high-resolution mapping systems. Stimulation maneuvers, on the other hand, may alter the AT into another arrhythmia [22,23]. Macro-reentry tachycardias were classified most commonly as roof-dependent or peri-mitral and treated by an anatomical approach. Takigawa et al. introduced the practical isthmus, usually the narrowest bridge of conductive tissue between scars or anatomic obstacles, as the easiest site for ablation [24]. Another possible ablation target is the slow conducting area in the mid-diastole of the excitation, often referred to as the critical isthmus $[25,26]$.

In this type of arrhythmia, the patient cohort is usually very heterogeneous. This study wanted to create comparable outcomes by applying predefined ablation strategies, depending on the type of reentry and the number of critical isthmuses. The primary objectives were the 12-month success and mean long-term success rates.

\section{Materials and Methods}

\subsection{Study Design}

This study was designed as a prospective, single group study. Inclusion criteria were sustaining left AT in patients with a history of AF and prior pulmonary vein isolation (PVI). Exclusion criteria were (1) an age younger than 18 years and (2) any contraindication to $C A$. The patients were treated with a substrate-based individualized ablation protocol (Figure 1) and followed up every six months. The study was conducted in accordance with the provisions of the Declaration of Helsinki and was approved by the ethics committee of the University of Stuttgart, Germany. The electrophysiological study and mapping was performed with the Rhythmia HDx ${ }^{\mathrm{TM}}$ Mapping System (Version 4.0.1.3). The electrophysiological (EP) study was conducted in accordance with the current guidelines [27]. ATs were sequentially mapped with the IntellaMap Orion ${ }^{\mathrm{TM}}$ multipolar basket catheter and Rhythmia HDx ${ }^{\mathrm{TM}}$ Mapping System. Local activation time (LAT) and voltage maps were acquired for all ATs. Electrogram annotation was performed automatically by the mapping system. After the map was completed, all efforts were redirected to track the propagating wavefront based on a local electrogram analysis, using the system's virtual roving probe, and to gain a detailed understanding of the reentry circuit [28-30]. All interpretations were based on the electrograms recorded with the IntellaMap Orion ${ }^{\mathrm{TM}}$ catheter. If the cycle length was fully covered, a reentry mechanism was assumed. Based on the discussed AT mechanisms at the time of the study initiation, an AT was predefined as (i) macro-reentry if the dominant path looped around an anatomical obstacle. Reentries which did not incorporate an anatomical obstacle were classified as (ii) localized reentries and were further differentiated into small-area reentries or micro-reentries. A (ii,a) small-area reentry was defined as a propagation around a dense scar area or a line with double potentials, not incorporating an anatomical obstacle. For (ii,b) a micro-reentry, the encircled area is reduced to a single spot. Centrifugal activation from a distinct focal source covering only part of the cycle length was considered as (iii) a focal AT [28,29,31]. Stable, spiral activations were predefined as (iv) rotor [32]. 

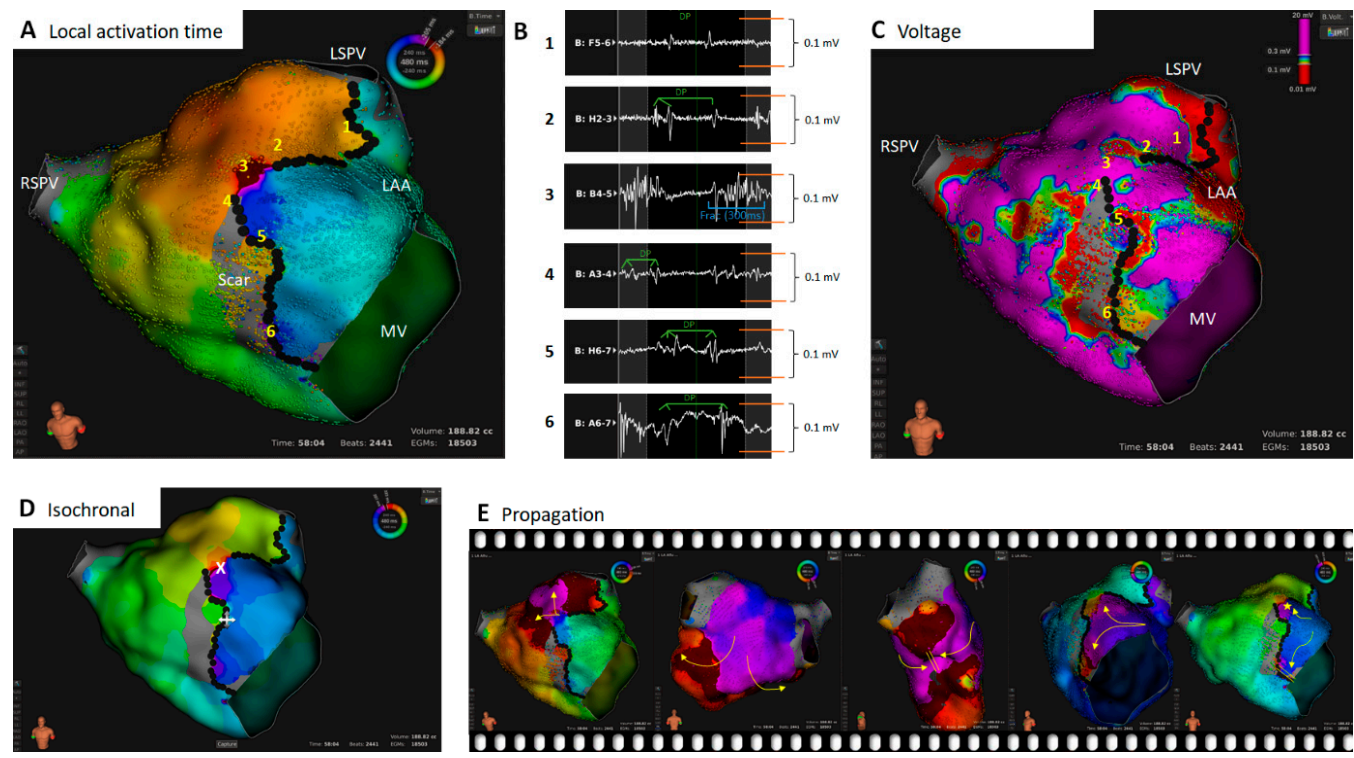

Figure 1. Patient with a persistent AF and a history of previous RF isolation of the PVs. The cycle length of the AT was $480 \mathrm{~ms}$. Shown is the ultra high-resolution map with the Rhythmia HDx ${ }^{\mathrm{TM}} 3 \mathrm{D}$ mapping system. (A): Local activation time map of the left atrium. Black dots represent locations with double potentials (DP). (B): Local bipolar electrograms. The numbers correspond to the locations in the LAT (A) and voltage map (C). (C): Voltage map; signal amplitude (sa) $>0.3 \mathrm{mV}=$ violet, sa $<0.1 \mathrm{mV}=$ red, $0.1 \mathrm{mV}>$ sa $<0.3 \mathrm{mV}=$ rainbow color, sa $<0.015 \mathrm{mV}=$ grey (noise level). Black dots represent locations with double potentials (DP). (D): LAT Isochronal, step size $30 \mathrm{~ms}$; critical isthmus marked with a white cross. (E): Propagation maps. Summary: The patient presented a macro-reentry AT with one critical isthmus. The dominant reentry propagated across the roof around the left PVs (A,E). A large scar area at the anterior wall was striking $(\mathbf{C})$. The isochronal map showed slow conduction at the upper and lower end of the scar area (D). Based on the voltage map (C), it was not feasible to delineate the distinct borders of the slow conducting area. A review of the electrograms revealed double potentials in areas 1 and 2 (B). However, another breakthrough at the lower end of the low voltage area appeared to be possible. Again, an electrogram review revealed double potentials in areas 4-6 (B). Of note: all signals which had two peaks within one AT cycle and were separated by an isoelectric line were classified as double potentials. This was also true if the single peaks were very close together or fractionated. A highly fractionated electrogram was found in area 3. Therefore, this spot was defined as a critical isthmus. The ablation strategy was a critical isthmus ablation only, which terminated the AT during the first RF delivery. Abbreviations: LSPV = left superior pulmonary vein; RSPV = right superior pulmonary vein; $\mathrm{MV}=$ mitral valve; $\mathrm{LAA}=$ left atrial appendage; $\mathrm{DP}=$ double potential.

\subsection{Catheter Ablation Approaches}

Reentries were ranked by the size of the pathway, indicating its stability. Microreentries, focal sources, or rotors underwent a manual EGM analysis to rule out visualization artefacts. If the driving mechanism was found to be reentry, the propagating wavefront was further analyzed for isthmus areas (Figure 1). Such narrow, slow conducting bridges are always bordered by adjacent scar areas, lines of block, or anatomical structures, electrocardiographically mainly represented by double potentials. Within these areas, the slowest conduction presenting the highest fractionation of the electrogram was defined as the critical isthmus. The primary aim of the ablation was to restore SR with a minimum of ablated tissue. The primary ablation strategy was to target the AT first, even if PV reconnection was discovered, to prove the hypothesis of the underlying mechanism. The distribution of the ablation strategies is outlined in Figure 2. For macro-reentries, areas with a reduced conduction velocity, fractionation, and double potentials were highlighted within the dominant circuit. The critical isthmus was defined as described above. If only 
one critical isthmus was present, a critical isthmus ablation was the sole ablation strategy. In cases with more than one critical isthmus, the ablation was extended to an individual line, equivalent to the Takigawa approach. The individual line aimed to connect the critical isthmus, via areas of slow conduction, to a line while ablating as little healthy tissue as possible and finally anchoring each end to an anatomical structure. If no slow conducting areas were found within the dominant circuit, a standard line was performed. A roof line, a mitral isthmus line [33], and a modified anterior line [34,35] were considered suitable standard lines. A proof of bidirectional block was mandatory for all lines. Localized reentries were treated either with critical isthmus ablation or an individual line, according to the protocol (Figure 2). The voltage map was only used supportively in theory-building. In the case of small-area reentries presenting with two or more critical isthmuses, it was left to the physician's discretion to choose the first ablation target. Subsequently, all additional critical isthmuses were ablated. Micro-reentries were locally ablated at their critical isthmus, including an additional ablation of fractionated electrograms in the immediate vicinity. Focal tachycardias were ablated at the site of the earliest bipolar and unipolar activation. The strategy for rotors was to ablate a slightly larger, local area at the earliest activated area for at least 30-60 s.

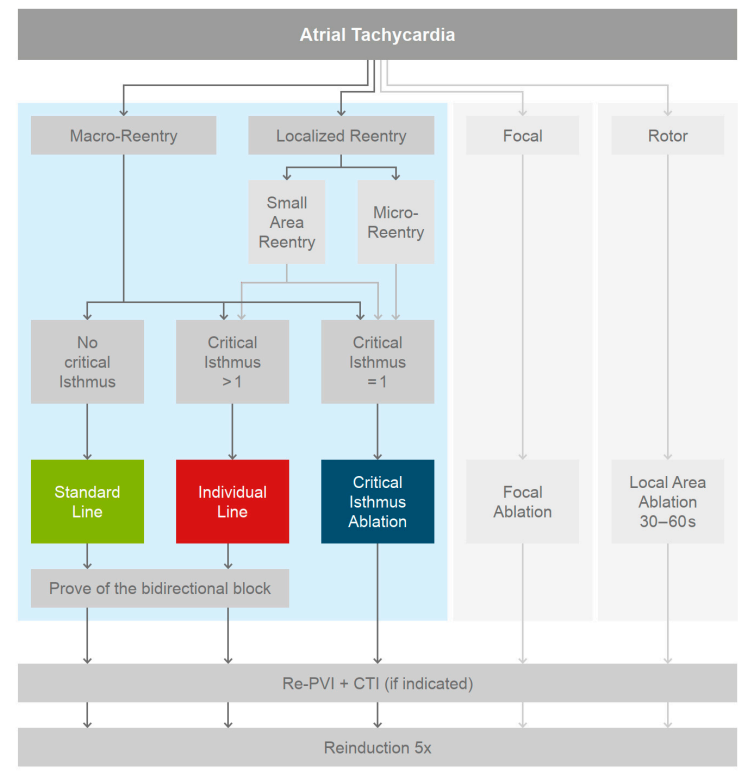

Figure 2. PVI = pulmonary vein isolation; $\mathrm{CTI}=$ cavotricuspid isthmus. Macro-reentry $=$ circular activation around an anatomical obstacle. Localized reentry = circular reentry not involving an anatomical structure but around a spot presenting double potential (micro-reentry) or around an area or line of block (small-area reentry). Focal $=$ true focal automatisms not related to structurally altered tissue, with a centrifugal excitation covering only part of the cycle length. Rotor $=$ spiral excitation wavefronts.

\subsection{Further Ablation Strategies and Reinduction}

After completion of the primary ablation strategy, all PV gaps were closed. Additionally, the cavotricuspid isthmus was ablated if common-type atrial flutter was suspected in the medical history. Repeated decremental atrial pacing of $<200 \mathrm{~ms}$ was performed five times to test for non-inducibility. All inducible sustaining ATs were mapped and ablated using the same approach. If AF was induced, external cardioversion was performed. Ablation was performed using the Directsense ${ }^{\mathrm{TM}}$ technology (Boston Scientific, Charlestown, MA, USA) [33,36] in conjunction with the HAT 500 RF-generator (Osypka, Rheinfelden, Germany). 


\subsection{Study Endpoints and Follow-Up}

The primary endpoint was clinical success after 12 months and long-term success. Secondary endpoints comprised (1) termination into SR overall, (2) termination into SR upon the primary ablation strategy as a proof of concept that the AT mechanism was completely decrypted, (3) time to termination in SR, and (4) the non-inducibility of any AT/AF. Follow-up was performed every 6 months in our outpatient clinic, including a medical history, 12-lead ECG, and $24 \mathrm{~h}$ Holter ECG, as well as a detailed interrogation for symptoms indicating possible AF or AT recurrence. In the case of a normal Holter ECG but reported arrhythmia episodes, patients received 7-day Holter-ECG monitoring to detect short arrhythmia episodes. The study did not include a blanking period. In the case of the recurrence of an incessant AT, a redo procedure was scheduled as soon as possible. Single-procedure and multi-procedure success was defined as the freedom from any AT after the index and the last ablation procedure, respectively.

\subsection{Statistical Analysis}

Variables are displayed as means with standard deviation. A Student's t-test was used for the comparison of patients. Freedom from any arrhythmia was quantified by the Kaplan-Meier estimator. A $p$-Value of $<0.05$ was considered statistically significant. Analyses were performed using Excel Version 2201, Matlab 2021b, or R Version 3.3.2.

\section{Results}

\subsection{Baseline Characteristics}

A total of 35 cases of incessant AT were included in the study. According to the inclusion criteria, all patients had a history of AF and had undergone previous PVI. The baseline characteristics are shown in Table 1 . On average, the patients had $2 \pm 1$ preablations in the left atrium, and the mean time to last left atrial ablation was $18 \pm 23$ months. With respect to all pre-procedures, $10(29 \%)$ had a PVI only, and $25(71 \%)$ had a previous PVI with additional substrate modification of the left atrium. A total of $13(37 \%)$ had, in addition, previous ablation due to a left AT. In the PVI-only group, $36 \%$ were pre-ablated with cryo energy. A total of $43 \%$ had undergone CTI ablation.

\subsection{Investigated Mechanisms of the Tachycardia}

A total of 54 tachycardias were mapped and ablated, with a maximum of four maps per patient. For the detailed analysis and calculations, only the map of the clinical AT was considered. Macro-reentries were present in $86 \%$ of the patients. Out of these, $42 \%(18 / 31)$ involved the mitral annulus, and 58\% involved the left atrial roof. Reentries encircling the ipsilateral PVs of each side were counted as roof-dependent. A total of $14 \%$ presented with a localized reentry. Of the localized reentries, one was classified as a small-area reentry and three as micro-reentries. Focal activations and rotors were not found in this study. The dominant reentry could be defined in $94 \%$ of the patients. In two patients, a dominant pathway could not be delineated. In both cases, the cycle length was completely covered by recorded electrogram activity, and mid-diastolic fractionated electrograms were found around a tiny area with double potentials. They were therefore classified as micro-reentries. A critical isthmus could be identified in $89 \%$ of patients. All critical isthmuses were found in areas with a reduced conduction velocity and low voltage. Fractionated electrograms in these regions could be found, with an average duration of $150 \pm 48 \mathrm{~ms}$ and voltage of $0.15 \pm 0.14 \mathrm{mV}$. The shortest electrogram measured $81 \mathrm{~ms}$, the longest was $234 \mathrm{~ms}$, and the minimal voltage was $0.04 \mathrm{mV}$. Out of the 31 macro reentries, $7(22 \%)$ were treated using a standard line (modified anterior line: $n=5$; roof line: $n=2), 13(42 \%)$ received an individual line, and $11(36 \%)$ had a critical isthmus ablation. In three patients with perimitral flutter, a rather broad slow conducting region was found anterior of the left atrial appendage without the presence of double potentials or a dense scar. As this region was located at the site of a modified anterior line, this line was chosen as an ablation strategy. Only one AT was found to be related to a gap in the previous PV line. This AT presented with two 
gaps, marking the entry and exit through the PV area, and it was therefore classified as a small area.

Table 1. Baseline characteristics.

\begin{tabular}{|c|c|}
\hline $\begin{array}{l}\text { Baseline Characteristics } \\
\text { Index Procedure }\end{array}$ & $\begin{array}{c}\text { Mean } \pm \text { SD or Total }(\%) \\
n=35\end{array}$ \\
\hline Age (years) & $65 \pm 7$ \\
\hline Gender, female $(n)$ & $12(40 \%)$ \\
\hline Hypertension $(n)$ & $26(87 \%)$ \\
\hline CAD $(n)$ & $8(27 \%)$ \\
\hline Diabetes mellitus $(n)$ & $3(10 \%)$ \\
\hline LVEF $<50 \%$ & $7(20 \%)$ \\
\hline CHA2DS2-VASc & $2 \pm 1$ \\
\hline LA size $(\mathrm{mm})$ & $43 \pm 6$ \\
\hline LA area $\left(\mathrm{cm}^{2}\right)$ & $23 \pm 5$ \\
\hline \multicolumn{2}{|l|}{ AAD } \\
\hline Betablocker $(n)$ & $21(60 \%)$ \\
\hline Amiodarone $(n)$ & $6(17 \%)$ \\
\hline Other $(n)$ & $0(0 \%)$ \\
\hline Number of prior left LA procedures $(n)$ & $2 \pm 1$ \\
\hline Time since last LA ablation (months) & $18 \pm 23$ \\
\hline Prior LA-AT ablation $(n)$ & $13(37 \%)$ \\
\hline \multicolumn{2}{|l|}{ Prior LA ablation approaches $(n)$} \\
\hline PVI only $(n)$ & $10(29 \%)$ \\
\hline PVI $+\operatorname{CFAE}(n)$ & $5(14 \%)$ \\
\hline PVI + LA-Lines $(n)$ & $9(26 \%)$ \\
\hline PVI + CFAE + Lines $(n)$ & $8(23 \%)$ \\
\hline PVI + Rotor $(n)$ & $3(9 \%)$ \\
\hline Prior CTI ablation $(n)$ & $15(43 \%)$ \\
\hline
\end{tabular}

CAD = coronary artery disease; $\mathrm{LVEF}=$ left ventricular ejection fraction; LA = left atrium; AAD = antiarrhythmic drugs; $\mathrm{AT}=$ atrial tachycardia; $\mathrm{PVI}=$ pulmonary vein isolation; $\mathrm{CFAE}=$ complex fractionated atrial electrograms; $\mathrm{CTI}=$ cavotricuspid isthmus. The AT was terminated immediately at the entry. The final ablation concept was the restoration of the completeness of the PV line with the closure of all gaps. The three micro-reentries were treated with a critical isthmus ablation, including the ablation of fractionated electrograms in the immediate vicinity.

\subsection{Primary Endpoint}

The multi-procedure success (1.14 procedures per patient) for freedom from any AT at 12 months after the last procedure was 90 . The single procedure success for freedom from any AT was $80 \%$. After a mean follow-up time of $30 \pm 13$ months, the multi-procedure success rate (1.14 procedures per patient) for the freedom from any AT was $77 \%$, and the single procedure success rate was $65 \%$ (Figure 3 ). The success rate of the critical isthmus approach was the highest, with $85 \%$, followed by individual line $(60 \%)$ and standard line $(57 \%)$, as illustrated in Figure 4.

\subsection{Secondary Endpoints}

\subsubsection{Termination into SR during CA}

The first secondary endpoint, the termination into SR during CA, could be reached in all patients. The second secondary endpoint, the termination into a sinus rhythm upon the first ablation strategy, was reached in 32 (91\%) of the patients.

During radiofrequency (RF)-energy delivery, the conversion to other AT forms was observed in three patients. Those ATs were mapped and ablated as described above. Conversion to AF did not occur. Procedural data are displayed in Table 2. The total procedure time was $193 \pm 57 \mathrm{~min}$, the mean fluoroscopy time was $12 \pm 8 \mathrm{~min}$, the mean fluoroscopy dosage was $621 \pm 555 \mathrm{cGycm}^{2}$, and the mean total RF time was $24 \pm 16 \mathrm{~min}$. A peculiarity of this study was that a major effort was spent on a high, homogeneously 
distributed point density in all areas of the map and on a detailed post-analysis with manual annotation of the electrograms. The mean mapping time was $28 \pm 10 \mathrm{~min}$.

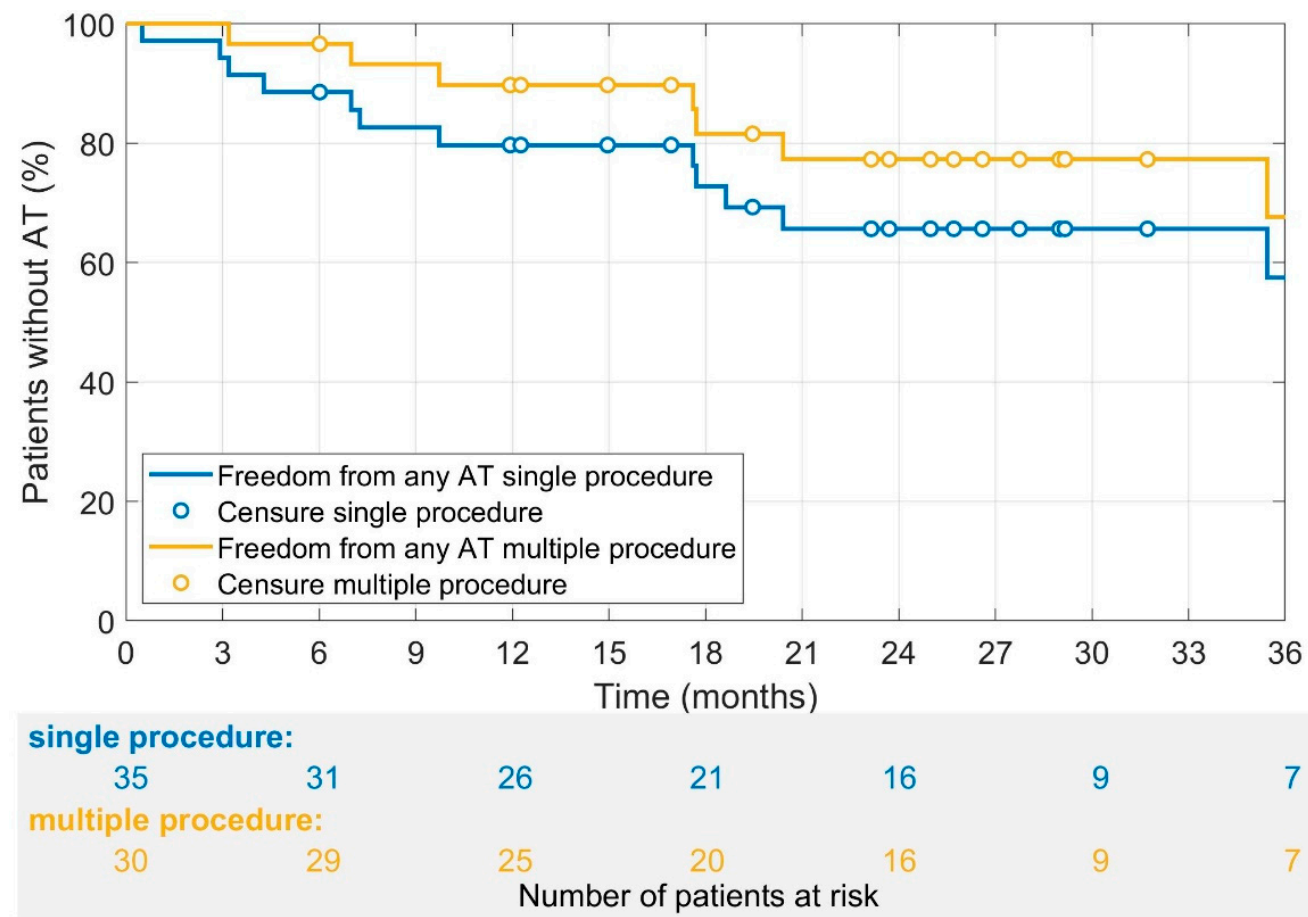

Figure 3. A Kaplan-Meier analysis for the freedom from any AT for multiple and single procedures per patient. The mean follow-up time was $30 \pm 13$ months for multiple procedures. Censored cases were indicated with yellow and blue circles. No blanking period was defined. The 12- and 24-month probability of freedom from any AT was $80 \%$ and $65 \%$ for single procedure and $90 \%$ and $77 \%$ for multiple procedures, respectively.

\section{Success Rates by Strategy}

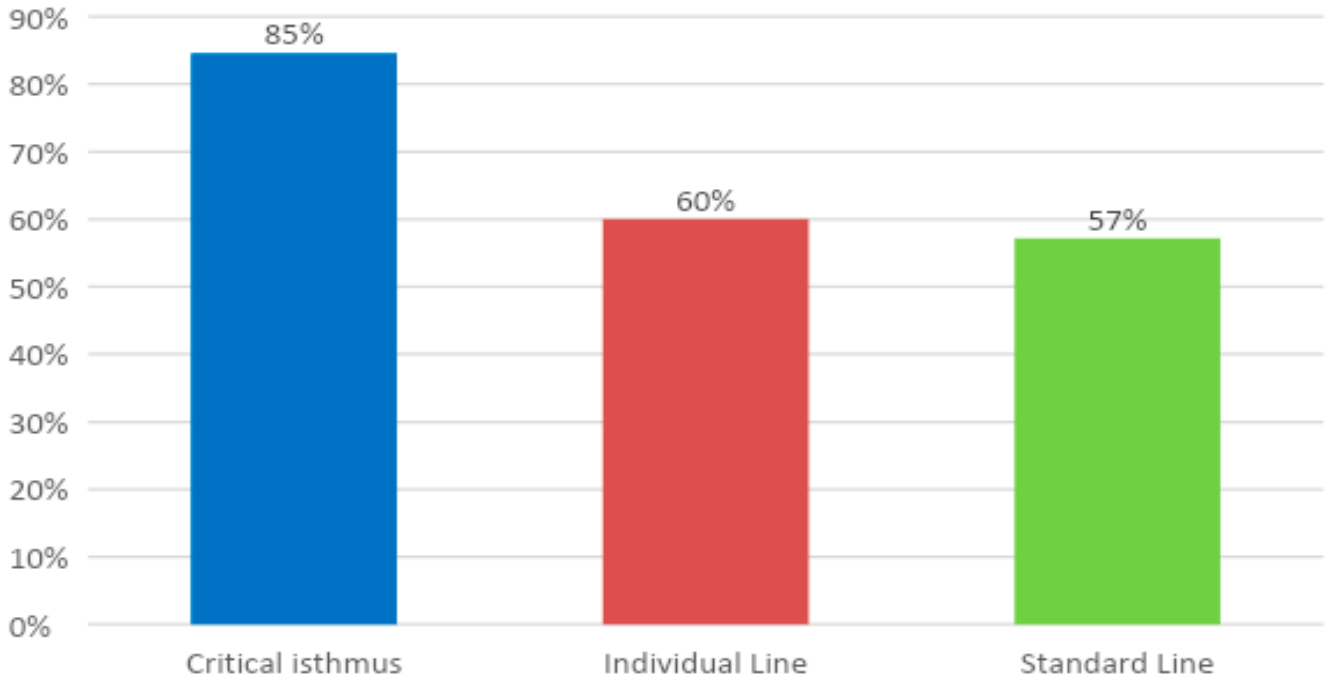

Figure 4. Clinical success dependent on the ablation strategy. Multi-procedure success after a mean follow-up time of 30 months. Differences were not significant. (Pearson Chi Square $p=0.28$ and $p=0.12$ when comparing critical isthmus vs. other lines). 
Table 2. Procedural Data.

\begin{tabular}{|c|c|}
\hline Procedural Data & $n=35$ \\
\hline Termination in SR $(n)$ & $35(100 \%)$ \\
\hline Termination in SR upon 1st ablation strategy $(n)$ & $32(91 \%)$ \\
\hline Total RF time (min) & $24 \pm 16$ \\
\hline Total procedure time (min) & $193 \pm 57$ \\
\hline Total fluoro time (min) & $12 \pm 8$ \\
\hline Total fluoro dosage (cGycm2) & $621 \pm 555$ \\
\hline Non-inducibility $(n)$ & $30(86 \%)$ \\
\hline Follow-up time (months) & $30 \pm 13$ \\
\hline Complications $(n)$ & $2(6 \%)$ \\
\hline Minor & $2(6 \%)$ \\
\hline Major & $0(0 \%)$ \\
\hline \multicolumn{2}{|l|}{ Ablation strategy $(n)$} \\
\hline Standard line & $7(20 \%)$ \\
\hline Individual line & $15(43 \%)$ \\
\hline Critical isthmus & $13(37 \%)$ \\
\hline Focal & $0(0 \%)$ \\
\hline Local area & $0(0 \%)$ \\
\hline Total time to SR (min) & $21 \pm 57$ \\
\hline Standard line & $57 \pm 100$ \\
\hline Individual line & $22 \pm 49$ \\
\hline Critical isthmus & $1 \pm 1$ \\
\hline RF time to SR (min) & $5 \pm 9$ \\
\hline Standard line & $14 \pm 12$ \\
\hline Individual line & $5 \pm 9$ \\
\hline Critical isthmus & $1 \pm 1$ \\
\hline Total RF applications to SR $(n)$ & $6 \pm 9$ \\
\hline Standard line & $16 \pm 13$ \\
\hline Individual line & $6 \pm 9$ \\
\hline Critical isthmus & $1 \pm 1$ \\
\hline
\end{tabular}

\subsubsection{Time to Termination}

A total of $32(91 \%)$ patients terminated in SR upon the first ablation strategy. In total, $100 \%$ terminated in SR during RF-delivery. The RF application time to restore SR differed between groups. It was significantly shorter in critical isthmus strategies $(1 \pm 1 \mathrm{~min})$ compared to individual line $(5 \pm 9 \mathrm{~min})$ and standard line $(14 \pm 12 \mathrm{~min})(p<0.01)$ strategies. The number of RF applications to restore SR was $1 \pm 1,6 \pm 9$, and $16 \pm 13$ for critical isthmus, individual line, and standard line, respectively. The total RF time was also significantly shorter in critical isthmus strategies (12 $\pm 11 \mathrm{~min})$ compared to individual line $(24 \pm 16 \mathrm{~min})$ and standard line $(32 \pm 12 \mathrm{~min}$ ) approaches $(p<0.01)$ (see Figure 5$)$. The total $\mathrm{RF}$ time included all additional ablations. The analysis of the total time to SR showed a trend towards shorter critical isthmus strategies $(1 \pm 1 \mathrm{~min})$ compared to individual line $(22 \pm 49 \mathrm{~min})$ and standard line approaches $(57 \pm 100 \mathrm{~min})(p=0.10)$. 
A: Radiofrequency Time to SR

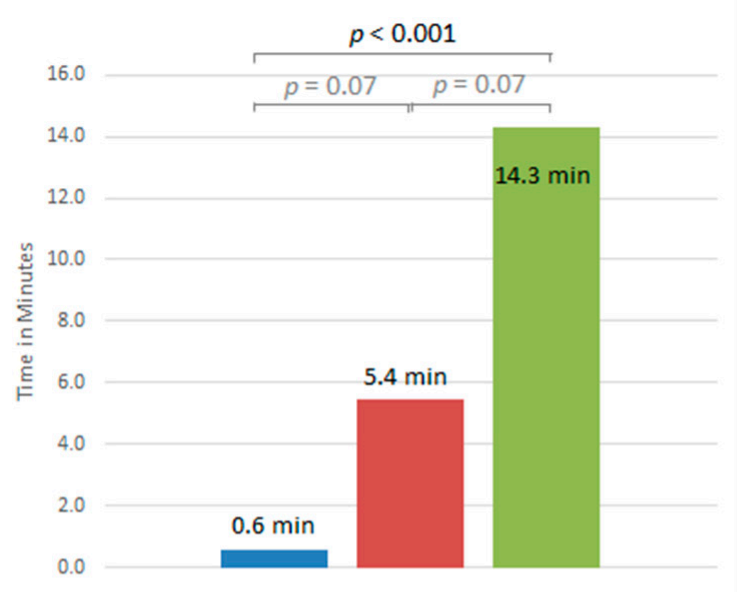

C: Total RF Time

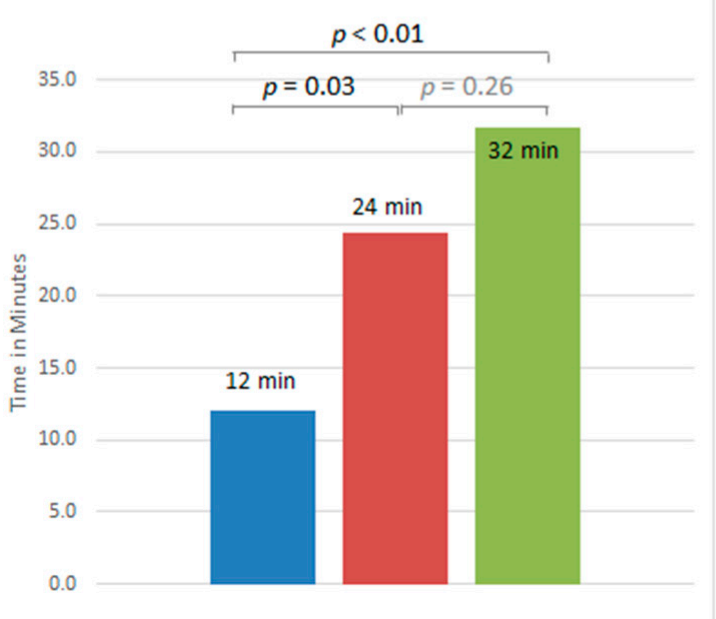

B: Total Time to SR

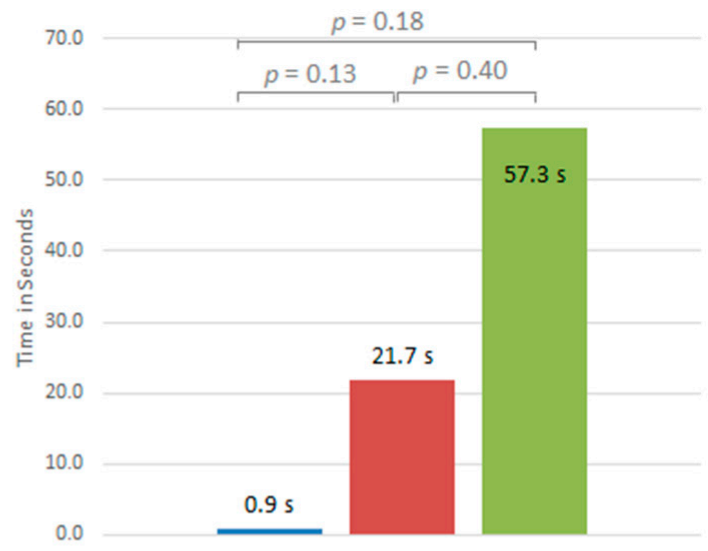

Figure 5. (A): RF time from the start of the first ablation until the termination in SR. For critical isthmus, individual line, and standard line, the RF time was $1 \pm 1 \mathrm{~min}, 5 \pm 9 \mathrm{~min}$, and $14 \pm 12 \mathrm{~min}$, respectively. Standard lines took significantly longer compared to critical isthmus ablation $(p<0.001)$. (B): The total time to SR was $1 \pm 1 \mathrm{~min}, 22 \pm 49 \mathrm{~min}$, and $57 \pm 100 \mathrm{~min}$, respectively. Differences were statistically not significant. (C): The total RF time, including the primary strategy and all additional ablations (i.e., achievement of bidirectional block, ablation of other critical isthmus, or fractionated potentials; re-PVI; and CTI ablation), was $12 \pm 11 \mathrm{~min}, 24 \pm 15 \mathrm{~min}$, and $32 \pm 12 \mathrm{~min}$, respectively. Standard lines and individual lines took significantly longer than critical isthmus ablation. $\mathrm{RF}=$ Radiofrequency; $\mathrm{SR}=$ Sinus rhythm.

\subsubsection{Non-Inducibility of Any Type of Arrhythmia}

After restoring the sinus rhythm and completing the AT ablation, re-PVI was performed if indicated. PVs were reconnected in 20 patients $(57 \%)$. A total of 2.4 PVs per patient presented PV potentials. All PVs could be successfully isolated by gap ablation on the previous ablation line, proven by entry block testing. In 7 (20\%) patients, additional CTI ablation was performed. After the ablation, in 30 out of the 35 cases (86\%), non-inducibility of any type of arrhythmia could be demonstrated (Table 3). The mean follow-up time was $30 \pm 13$ months. During the follow-up time, five patients needed a redo procedure due to the recurrence of AT. 
Table 3. Mapping Data.

\begin{tabular}{cc}
\hline Mapping Data & $n=35$ \\
\hline AT cycle length $(\mathrm{ms})$ & $297 \pm 86$ \\
P-wave duration in AT (ms) & $117 \pm 32$ \\
ATs mapped $(n) /$ patient & $1 \pm 1$ \\
Points per map $(n)$ & $20,773 \pm 9748$ \\
LA volume $(\mathrm{mL})$ & $188 \pm 48$ \\
\hline Mapping time $(\mathrm{min})$ & $27 \pm 10$ \\
Dominant reentry defined $(n)$ & $33(94 \%)$ \\
Critical isthmus present $(n)$ & $31(89 \%)$ \\
Type of AT $(n)$ & $31(88 \%)$ \\
Macro & $1(3 \%)$ \\
Small area & $3(9 \%)$ \\
Micro & $0(0.0 \%)$ \\
Focal & $0(0.0 \%)$ \\
Rotor & $47(33 \%)$ \\
PV-reconnection $(n)$ & $1.6 \pm 1$ \\
\hline PV-reconnection per patient $(n)$ & atrial tachycardia; LA = left atrium; WOI = windows of interest; PV = pulmonary vein.
\end{tabular}

\subsubsection{Learning Curve}

The learning curve analysis showed significant differences in the success rates and procedure time. Freedom from any AT was $20 \%$ for the first 5 procedures, as opposed to $80 \%$ for the last 5 procedures. Total procedure time was $251 \pm 77 \mathrm{~min}$ and $149 \pm 40 \mathrm{~min}$ $(p=0.04)$, respectively.

\section{Discussion}

The demand for a more successful treatment of AT has been rising as a consequence of increasing numbers of cardiac surgery and catheter-based ablation of AF [37]. However, recurrent AT in pre-ablated atrial tissue is challenging to map and interpret, due to an incomplete understanding of the mechanisms as a consequence of areas of scar, low amplitude electrograms, and a multiplicity of arrhythmias within a given patient [38]. According to van Marion et al., the electro-pathological substrate remains poorly understood, and hence, defining the optimal approach per individual patient is challenging, whilst electropathology cannot be evaluated [39]. With the advent of high-resolution 3D mapping, some of these obstacles might be overcome by identifying conduction circuits in more granular detail [25]. However, even if the AT has been completely mapped using high resolution 3D mapping systems, the classification varies within the EP community. Differences have mainly been described concerning macro-reentry and focal tachycardias, leading to different incidences in the literature $[17,18,40]$. We studied 35 recurrent AT patients with the high-resolution 3D electro-anatomical mapping system Rhythmia HDx ${ }^{\mathrm{TM}}$ in order to best confirm the hypothesized advantages of a standardized, algorithm-based ablation approach for complex arrhythmias resulting from potential benefits of complete mechanism traceability. Despite the availability of high-resolution 3D electro-anatomical mapping since several years ago, there is still no standardized terminology in the literature for whether macro-reentry tachycardias circle around a central obstacle of a certain size [24,41] or around an anatomical structure [42]. In this study, tachycardias circling around an anatomical structure were classified as macro-reentry. The term "focal" was used in some studies [40-42] for both micro-reentry and true focal automatisms, making it difficult to compare reported incidences and success rates. Non-reentrant focal tachycardias may have so far not been mapped accurately enough and have therefore been difficult to differentiate from micro-reentry tachycardias [43]. In our study, micro-reentries were separated from focal tachycardias by a manual analysis of local electrograms. We identified $86 \%$ macroand $14 \%$ micro-reentry mechanisms. Focal automatisms or rotors could not be detected, with respect to the definition used in this study. Hence, with the new high-resolution 
3D mapping systems, the focal or centrifugal origin hypothesis might be challenged for post-PVI patients. Decisive for our ablation approach was the determination of the dominant reentry and the number of critical isthmuses contained. Macro-reentries were only designated as such if they circled around an anatomical obstacle. Standard lines were only used in macro-reentries if no critical isthmus and no area of slow conduction could be found within the dominant reentry. Micro-reentry tachycardias were distinguished from focal automatisms by means of a manual signal analysis and were ablated accordingly. All other tachycardias were treated by an individual approach creating an ablation line between two non-conducting anatomical structures, containing the critical isthmus and slow conducting areas of the dominant reentry and passing through as little healthy tissue as possible. This strategy combines the tailored approach [24] and the critical isthmus ablation $[25,26]$. The primary intent of the study was to terminate the AT as fast as and with as little damage to healthy tissue as possible in order to achieve long-term benefits for the patients with left atrial tachycardia, following catheter ablation of atrial fibrillation. Non-inducibility was demonstrated after the ablation of all concomitant ATs. Derval et al. mainly used standard lines for ablation and reported $54 \%$ freedom from any AT after 12 months in 111 post-PVI patients in a comparative study [42]. The high percentage of a previous substrate modification and the proportion of macro-reentry tachycardias was comparable to our study population. The number of right atrial ablations was higher in the comparative dataset [42]. In our cohort, the success rate was $82 \%$ for single and $90 \%$ for multiple procedures using the individualized approach. Comparing results specifically for macro-reentry tachycardias, the ratio of anatomical to individual lines was $22 \% / 78 \%$ in our data, as compared to $89 \% / 11 \%$ in the comparative cohort. In our study, anatomical lines were only applied if the macro-reentry tachycardia showed no individual substrate or arrhythmia-specific regional conduction slowing. For peri-mitral ATs, we applied, in all cases, a modified anterior line, whereas the mitral isthmus line was predominantly used in the comparative study [42]. Takigawa et al. also introduced a tailored approach [24]. They defined the practical isthmus as the narrowest bridge of conducting tissue between scars or anatomical obstacles, which was often found to be different from the anatomical isthmus. ATs could be terminated in $74 \%$, and the success rate after 6 months was $74 \%$. Adapting and further developing this approach, Jungen et al. reported $73 \%$ freedom from AT after seven months and a mean of 1.4 procedures per patient [41].

In this study, the different ablation strategies were used according to the type of reentry and the number of critical isthmuses. The individual line combines the tailored approach [24] and critical isthmus ablation $[25,26]$ in those patients with more than one critical isthmus. All ATs could be terminated into SR, and 91\% terminated into SR upon the first ablation strategy. A bidirectional block was confirmed for all lines by stimulation. Freedom from AT could be demonstrated in $80 \%$ and $57 \%$ for single and $90 \%$ and $77 \%$ for multiple procedures after 12 and 30 months and a mean of 1.14 procedures, respectively. Patients receiving a critical isthmus ablation showed the best outcome. The average ablation time in this group to restore SR was $1 \mathrm{~min}$, with a clinical success rate of $85 \%$ after a mean follow-up time of 30 months. The individual line was ranked second, while the anatomical line brought up the rear. However, the short-cut conclusion, that a critical isthmus ablation is superior to other strategies, was not valid, as the applied ablation strategy was chosen based on the underlying individual substrate. Rather the existing substrate was clearly defined and less complex in patients receiving a critical isthmus ablation. For patients who received a standard line due to a lack of areas with significantly reduced conduction velocity, the natural question arises of whether a pathological substrate might have not been recognized due to the current limitations of the available technologies. However, the acute and, in particular, 12-month and mean long-term success rates of this study reinforce the need of individual approaches in these complex patients. Furthermore, our study, to our knowledge, for the first time, covered a midterm follow-up of up to 41 months. While achieving similar acute results after 30 days like Schaeffer et al. [40], the newly applied specific individualized approach led to a success rate of $83 \%$ after one year, with relapses 
being associated with a higher age. We could also report a mid-term freedom from any AT rate of $65 \%$ for single and $77 \%$ for multiple procedures after a mean follow-up time of 30 months in these complex patients. The multi-procedure success rate showed only a slight improvement, which seemed unexpected but might be caused by the extremely diseased atria in these patients with multiple ablations and progressed substrate development.

\section{Limitations}

This was a single-center investigation, with a high selective patient cohort of low numbers. Only patients with ongoing stable AT and previous PVI were included in the study. It is therefore worth discussing if the findings and success rates could be transferred to a wider AT population. To overcome the limitations of the visualization of the 3D mapping system due to electrograms presenting with very low voltage, a manual electrogram analysis, to define the critical parts of the dominant reentry, was performed in this study. It is questionable if such an effort can be implemented in everyday clinical practice and whether inter-observer variabilities may lead to different results. Therefore, there is a demand for automatic algorithms to shorten, standardize, and simplify the analysis [44] and to prove the practicability of this approach in larger multi-center studies. The lowest electrogram amplitude recorded in a critical isthmus measured $0.015 \mathrm{mV}$. This demonstrates the need for a high-resolution catheter and raises the question of whether even an improved signalto-noise ratio would add additional information to the understanding of the arrhythmia. Jungen et al. demonstrated high effectiveness and very low recurrence rates of CA in clinical tachycardia [41]. However, even if non-inducibility could be demonstrated at the end of the ablation, recurrencies of other arrhythmias were reported to be high. Therefore, an interesting question is whether computer simulation could help to identify further arrhythmia-specific substrates and, thus, could increase CA success rates [45].

\section{Conclusions}

With currently available ablation strategies, in combination with a predefined workflow based on the reentry mechanism and the number of critical isthmuses, a multiple procedure success rate of $90 \%$ for any AT recurrence at one-year could be reached. The more precisely the underlying substrate can be defined, the less ablation time is required. Tachycardia with a single critical isthmus can be ablated by critical isthmus ablation only, but the vast majority still require lines.

Author Contributions: Conceptualization, A.L.; Data curation, A.H.; Formal analysis, A.H. and B.B.; Funding acquisition, A.L.; Investigation, A.L.; Methodology, A.L. and A.H.; Writing-original draft, A.L. and B.B.; Writing-review and editing, K.S., L.U. and P.T. All authors have read and agreed to the published version of the manuscript.

Funding: This research received external funding by the German research society (Deutsche Forschungsgemeinschaft) under the number LU 2294/1-1.

Institutional Review Board Statement: The study was conducted according to the guidelines of the Declaration of Helsinki and approved by the Ethics Committee of the University of Stuttgart.

Informed Consent Statement: Informed consent was obtained from all subjects involved in the study.

Acknowledgments: We would like to thank Sigrun Müller for the excellent support in the data acquisition and Karin Etzold for the valuable help with the illustrations.

Conflicts of Interest: Luik has received speakers-fees from Boston Scientific, Biosense Webster, and Bristol-Myer Squibb. All other authors declare no conflicts of interests. The funders had no role in the design of the study; in the collection, analyses, or interpretation of data; in the writing of the manuscript; or in the decision to publish the results. 


\section{References}

1. Granada, J.; Uribe, W.; Chyou, P.-H.; Maassen, K.; Vierkant, R.; Smith, P.N.; Hayes, J.; Eaker, E.; Vidaillet, H. Incidence and predictors of atrial flutter in the general population. J. Am. Coll. Cardiol. 2000, 36, 2242-2246. [CrossRef]

2. $\quad$ Elesber, A.A.; Rosales, A.G.; Herges, R.M.; Shen, W.-K.; Moon, B.S.; Malouf, J.F.; Ammash, N.M.; Somers, V.; Hodge, D.O.; Gersh, B.J.; et al. Relapse and mortality following cardioversion of new-onset vs. recurrent atrial fibrillation and atrial flutter in the elderly. Eur. Hear. J. 2006, 27, 854-860. [CrossRef]

3. Gerstenfeld, E.P.; Callans, D.J.; Dixit, S.; Russo, A.M.; Nayak, H.; Lin, D.; Pulliam, W.; Siddique, S.; Marchlinski, F. Mechanisms of Organized Left Atrial Tachycardias Occurring After Pulmonary Vein Isolation. Circulation 2004, 110, 1351-1357. [CrossRef] [PubMed]

4. Gucuk Ipek, E.; Marine, J.E.; Habibi, M.; Chrispin, J.; Lima, J.; Rickard, J.; Spragg, D.; Zimmerman, S.L.; Zipunnikov, V.; Berger, R.; et al. Association of left atrial function with incident atypical atrial flutter after atrial fibrillation ablation. Heart Rhythm. 2016, 13, 391-398. [CrossRef] [PubMed]

5. Chugh, A.; Oral, H.; Lemola, K.; Hall, B.; Cheung, P.; Good, E.; Tamirisa, K.; Han, J.; Bogun, F.; Pelosi, F.; et al. Prevalence, mechanisms, and clinical significance of macroreentrant atrial tachycardia during and following left atrial ablation for atrial fibrillation. Hear. Rhythm 2005, 2, 464-471. [CrossRef]

6. Page, R.L.; Joglar, J.A.; Caldwell, M.A.; Calkins, H.; Conti, J.B.; Deal, B.J.; Estes, N.A.M.; Field, M.E.; Goldberger, Z.D.; Hammill, S.C.; et al. 2015 ACC/AHA/HRS guideline for the management of adult patients with supraventricular tachycardia: A report of the American College of Cardiology/American Heart Association Task Force on Clinical Practice Guidelines and the Heart Rhythm Society. Circulation 2016, 133, e506-e574. [CrossRef]

7. Ferrero de Loma-Osorio, Á.; Díaz-Infante, E.; Macías Gallego, A. Spanish Catheter Ablation Registry. 12th Official Report of the Spanish Society of Cardiology Working Group on Electrophysiology and Arrhythmias (2012). Revista Española Cardiología 2013, 66, 983-992. [CrossRef]

8. Baker, B.M.; Lindsay, B.D.; Bromberg, B.I.; Frazier, D.W.; Cain, M.E.; Smith, J.M. Catheter ablation of clinical intraatrial reentrant tachycardias resulting from previous atrial surgery: Localizing and transecting the critical isthmus. J. Am. Coll. Cardiol. 1996, 28, 411-417. [CrossRef]

9. Triedman, J.K.; Bergau, D.M.; Saul, J.; Epstein, M.R.; Walsh, E.P. Efficacy of Radiofrequency Ablation for Control of Intraatrial Reentrant Tachycardia in Patients With Congenital Heart Disease. J. Am. Coll. Cardiol. 1997, 30, 1032-1038. [CrossRef]

10. Chan, D.P.; Van Hare, G.F.; Mackall, J.A.; Carlson, M.D.; Waldo, A.L. Importance of Atrial Flutter Isthmus in Postoperative Intra-Atrial Reentrant Tachycardia. Circulation 2000, 102, 1283-1289. [CrossRef]

11. Jaïs, P.; Shah, D.C.; Haïssaguerre, M.; Hocini, M.; Tian Peng, J.; Takahashi, A.; Garrigue, S.; le Métayer, P.; Clémenty, J. Mapping and Ablation of Left Atrial Flutters. Circulation 2000, 101, 2928-2934. [CrossRef] [PubMed]

12. Akar, J.G.; Kok, L.C.; Haines, D.E.; DiMarco, J.P.; Mounsey, J. Coexistence of type I atrial flutter and intra-atrial re-entrant tachycardia in patients with surgically corrected congenital heart disease. J. Am. Coll. Cardiol. 2001, 38, 377-384. [CrossRef]

13. Nakagawa, H.; Shah, N.; Matsudaira, K.; Overholt, E.; Chandrasekaran, K.; Beckman, K.J.; Spector, P.; Calame, J.D.; Rao, A.; Otomo, K.; et al. Characterization of Reentrant Circuit in Macroreentrant Right Atrial Tachycardia After Surgical Repair of Congenital Heart Disease Isolated Channels Between Scars Allow “Focal” Ablation. Circulation 2001, 103, 699-709. [CrossRef] [PubMed]

14. Coffey, J.O.; D’avila, A.; Dukkipati, S.; Danik, S.B.; Gangireddy, S.R.; Koruth, J.S.; Miller, M.A.; Sager, S.J.; Eggert, C.A.; Reddy, V.Y Catheter ablation of scar-related atypical atrial flutter. Europace 2013, 15, 414-419. [CrossRef] [PubMed]

15. Stevenson, I.H.; Kistler, P.M.; Spence, S.J.; Vohra, J.K.; Sparks, P.B.; Morton, J.B.; Kalman, J.M. Scar-related right atrial macroreentrant tachycardia in patients without prioratrial surgery: Electroanatomic characterization and ablation outcome. Heart Rhythm 2005, 2, 594-601. [CrossRef]

16. Winkle, R.A.; Moskovitz, R.; Mead, R.H.; Engel, G.; Kong, M.H.; Fleming, W.; Patrawala, R.A. Ablation of atypical atrial flutters using ultra high density-activation sequence mapping. J. Interv. Card. Electrophysiol. 2017, 48, 177-184. [CrossRef]

17. Jaïs, P.; Hocini, M.; Weerasoryia, R.; Macle, L.; Scavee, C.; Raybaud, F.; Shah, D.C.; Clémenty, J.; Haïssaguerre, M. Atypical left atrial flutters. Kluwer Acad. Publ. 2002, 6, 371-377. [CrossRef]

18. Bun, S.-S.; Latcu, D.G.; Marchlinski, F.; Saoudi, N. Atrial flutter: More than just one of a kind. Eur. Heart J. 2015, 36, 2356-2363. [CrossRef]

19. Pedrinazzi, C.; Durin, O.; Mascioli, G.; Curnis, A.; Raddino, R.; Inama, G.; Cas, L.D. Atrial Flutter: From ECG to Electroanatomical 3D Mapping. Heart Int. 2006, 2. [CrossRef]

20. Sundaram, S.; Choe, W.; Jordan, J.R.; Mullins, N.; Boorman, C.; Kessler, E.J.; Nath, S. Catheter ablation of atypical atrial flutter: A novel 3D anatomic mapping approach to quickly localize and terminate atypical atrial flutter. J. Interv. Card. Electrophysiol. 2017, 49, 307-318. [CrossRef]

21. Patel, A.M.; d'Avila, A.; Neuzil, P.; Kim, S.J.; Mela, T.; Singh, J.P.; Ruskin, J.N.; Reddy, V.Y. Atrial tachycardia after ablation of persistent atrial fibrillation: Identification of the critical isthmus with a combination of multielectrode activation mapping and targeted entrainment mapping. Circ. Arrhythmia Electrophysiol. 2008, 1, 14-22. [CrossRef] [PubMed]

22. Ju, W.; Yang, B.; Chen, H.; Zhang, F.; Gu, K.; Yu, J.; Li, M.; Yang, G.; Cao, K.; Chen, M. Mapping of focal atrial tachycardia with an uninterpretable activation map after extensive atrial ablation: Tricks and tips. Circ. Arrhythmia Electrophysiol. 2014, 7, 598-604. [CrossRef] 
23. Schaeffer, B.; Stevenson, W.G. Entrainment mapping: Theoretical considerations and practical implementation. J. Cardiovasc. Electrophysiol. 2017, 29, 204-213. [CrossRef]

24. Takigawa, M.; Derval, N.; Frontera, A.; Martin, R.; Yamashita, S.; Cheniti, G.; Vlachos, K.; Thompson, N.; Kitamura, T.; Wolf, M.; et al. Revisiting anatomic macroreentrant tachycardia after atrial fibrillation ablation using ultrahigh-resolution mapping: Implications for ablation. Heart Rhythm. 2018, 15, 326-333. [CrossRef] [PubMed]

25. Lațcu, D.G.; Bun, S.-S.; Viera, F.; Delassi, T.; El Jamili, M.; Al Amoura, A.; Saoudi, N. Selection of Critical Isthmus in Scar-Related Atrial Tachycardia Using a New Automated Ultrahigh Resolution Mapping System. Circ. Arrhythmia Electrophysiol. 2017, 10, e004510. [CrossRef] [PubMed]

26. De Ponti, R.; Verlato, R.; Bertaglia, E.; Del Greco, M.; Fusco, A.; Bottoni, N.; Drago, F.; Sciarra, L.; Ometto, R.; Mantovan, R.; et al. Treatment of macro-re-entrant atrial tachycardia based on electroanatomic mapping: Identification and ablation of the middiastolic isthmus. Europace 2007, 9, 449-457. [CrossRef]

27. Kuck, K.; Böcker, D.; Chun, J.; Deneke, T.; Hindricks, G.; Hoffmann, E.; Piorkowski, C.; Willems, S. Qualitätskriterien zur Durchführung der Katheterablation von Vorhofflimmern. Kardiologe 2017, 11, 161-182. [CrossRef]

28. Jaïs, P.; Matsuo, S.; Knecht, S.; Weerasooriya, R.; Hocini, M.; Sacher, F.; Wright, M.; Nault, I.; Lellouche, N.; Klein, G.; et al. A Deductive Mapping Strategy for Atrial Tachycardia Following Atrial Fibrillation Ablation: Importance of Localized Reentry. J. Cardiovasc. Electrophysiol. 2009, 20, 480-491. [CrossRef]

29. Sanders, P.; Hocini, M.; Jaïs, P.; Hsu, L.-F.; Takahashi, Y.; Rotter, M.; Scavée, C.; Pasquié, J.-L.; Sacher, F.; Rostock, T.; et al Characterization of Focal Atrial Tachycardia Using High-Density Mapping. J. Am. Coll. Cardiol. 2005, 46, 2088-2099. [CrossRef]

30. Saoudi, N.; Cosio, F.; Waldo, A.E.A.; Chen, S.A.; Iesaka, Y.; Lesh, M.; Saksena, S.; Salerno, J.; Schoels, W. Classification of Atrial Flutter and Regular Atrial Tachycardia According to Electrophysiologic Mechanism and Anatomic Bases. J. Cardiovasc. Electrophysiol. 2001, 12, 852-866. [CrossRef]

31. Rostock, T.; Drewitz, I.; Steven, D.; Hoffmann, B.A.; Salukhe, T.V.; Bock, K.; Servatius, H.; Aydin, M.A.; Meinertz, T.; Willems, S. Characterization, Mapping, and Catheter Ablation of Recurrent Atrial Tachycardias After Stepwise Ablation of Long-Lasting Persistent Atrial Fibrillation. Circ. Arrhythmia Electrophysiol. 2010, 3, 160-169. [CrossRef] [PubMed]

32. Narayan, S.M.; Krummen, D.E. Targeting Stable Rotors to Treat Atrial Fibrillation. Arrhythm. Electrophysiol. Rev. 2012, 1, 34-38. [CrossRef] [PubMed]

33. Jaïs, P.; Hocini, M.; Hsu, L.-F.; Sanders, P.; Scavee, C.; Weerasooriya, R.; Macle, L.; Raybaud, F.; Garrigue, S.; Shah, D.C.; et al Technique and Results of Linear Ablation at the Mitral Isthmus. Circulation 2004, 110, 2996-3002. [CrossRef] [PubMed]

34. Tzeis, S.; Luik, A.; Jilek, C.; Schmitt, C.; Estner, H.L.; Wu, J.; Reents, T.; Fichtner, S.; Kolb, C.; Karch, M.R.; et al. The Modified Anterior Line: An Alternative Linear Lesion in Perimitral Flutter. J. Cardiovasc. Electrophysiol. 2010, 21, 665-670. [CrossRef] [PubMed]

35. Ammar, S.; Luik, A.; Hessling, G.; Bruhm, A.; Reents, T.; Semmler, V.; Buiatti, A.; Kathan, S.; Hofmann, M.; Kolb, C.; et al. Ablation of perimitral flutter: Acute and long-term success of the modified anterior line. Europace 2015, 17, 447-452. [CrossRef] [PubMed]

36. Das, M.; Luik, A.; Shepherd, E.; Sulkin, M.; Laughner, J.; Duffy, E.; Oesterlein, T.; Meyer, C.; Jais, P.; Duchateau, J.; et al. Local catheter impedance drop during pulmonary vein isolation predicts conduction block in patients with paroxysmal atrial fibrillation: Initial results of the LOCALIZE clinical trial. Europace 2020, 22, euaa162.249. Available online: https://academic.oup.com/ europace/article/22/Supplement_1/euaa162.249/5858672 (accessed on 18 October 2021). [CrossRef]

37. Munro, J.; Shen, W.-K.; Ru-Xing, W. A practical approach to atypical atrial flutter. J. Pract. Electrophysiol. 2015, $24,229-244$.

38. Ghannam, M.; Chugh, A. Utility of the Electrocardiogram in Mapping of Atrial Tachycardia Post-Atrial Fibrillation Ablation: Back to the Basics. JACC Clin. Electrophysiol. 2018, 4, 46-48. [CrossRef]

39. Van Marion, D.M.S.; Lanters, E.A.H.; Wiersma, M.; Allessie, M.A.; Brundel, B.B.J.J.M.; de Groot, N.M.S. Diagnosis and Therapy of Atrial Fibrillation: The Past, the Present and the Future. J. Atrial Fibrillation 2015, 8, 51-60.

40. Schaeffer, B.; Hoffmann, B.A.; Meyer, C.; Akbulak, R.; Moser, J.; Jularic, M.; Eickholt, C.; Nührich, J.M.; Kuklik, P.; Willems, S Characterization, Mapping, and Ablation of Complex Atrial Tachycardia: Initial Experience With a Novel Method of Ultra High-Density 3D Mapping. J. Cardiovasc. Electrophysiol. 2016, 27, 1139-1150. [CrossRef]

41. Jungen, C.; Akbulak, R.; Kahle, A.; Eickholt, C.; Schaeffer, B.; Scherschel, K.; Dinshaw, L.; Muenkler, P.; Schleberger, R.; Nies, M.; et al. Outcome after tailored catheter ablation of atrial tachycardia using ultra-high-density mapping. J. Cardiovasc. Electrophysiol. 2020, 31, 2645-2652. [CrossRef] [PubMed]

42. Derval, N.; Takigawa, M.; Frontera, A.; Mahida, S.; Konstantinos, V.; Denis, A.; Duchateau, J.; Pillois, X.; Yamashita, S.; Berte, B.; et al. Characterization of Complex Atrial Tachycardia in Patients With Previous Atrial Interventions Using HighResolution Mapping. JACC Clin. Electrophysiol. 2020, 6, 815-826. [CrossRef] [PubMed]

43. Ng, F.S.; Guerrero, F.; Luther, V.; Sikkel, M.; Lim, P.B. Microreentrant left atrial tachycardia circuit mapped with an ultra-highdensity mapping system. Heart Rhythm Case Rep. 2017, 3, 224-228. [CrossRef] [PubMed]

44. Oesterlein, T.G.; Loewe, A.; Lenis, G.; Luik, A.; Schmitt, C.; Doessel, O. Automatic Identification of Reentry Mechanisms and Critical Sites During Atrial Tachycardia by Analyzing Areas of Activity. IEEE Trans. Biomed. Eng. 2018, 65, 2334-2344. [CrossRef]

45. Loewe, A.; Poremba, E.; Oesterlein, T.; Luik, A.; Schmitt, C.; Seemann, G.; Dössel, O. Patient-Specific Identification of Atrial Flutter Vulnerability-A Computational Approach to Reveal Latent Reentry Pathways. Front. Physiol. 2019, 9, 1910. [CrossRef] 